\title{
Wave propagation in cross-linked random fiber networks
}

\section{Citation}

Babaee, Sahab, A. S. Shahsavari, Pai Wang, R. C. Picu, and Katia Bertoldi. 2015. “Wave Propagation in Cross-Linked Random Fiber Networks." Applied Physics Letters 107 (21)

(November 23): 211904. Portico. doi:10.1063/1.4936327.

\section{Published Version}

doi:doi.org/10.1063/1.4936327

\section{Permanent link}

http://nrs.harvard.edu/urn-3:HUL.InstRepos:27657495

\section{Terms of Use}

This article was downloaded from Harvard University's DASH repository, and is made available under the terms and conditions applicable to Other Posted Material, as set forth at http:// nrs.harvard.edu/urn-3:HUL.InstRepos:dash.current.terms-of-use\#LAA

\section{Share Your Story}

The Harvard community has made this article openly available.

Please share how this access benefits you. Submit a story.

Accessibility 


\section{AIP / Applied Physics Letters}

\section{Wave propagation in cross-linked random fiber networks}

Sahab Babaee, A. S. Shahsavari, Pai Wang, R. C. Picu, and Katia Bertoldi

Citation: Applied Physics Letters 107, 211904 (2015); doi: 10.1063/1.4936327

View online: http://dx.doi.org/10.1063/1.4936327

View Table of Contents: http://scitation.aip.org/content/aip/journal/apl/107/21?ver=pdfcov

Published by the AIP Publishing

\section{Articles you may be interested in}

Kink and kink-like waves in pre-stretched Mooney-Rivlin viscoelastic rods

AIP Advances 5, 087167 (2015); 10.1063/1.4929721

Tantalum and vanadium response to shock-wave loading at normal and elevated temperatures. Nonmonotonous decay of the elastic wave in vanadium

J. Appl. Phys. 115, 243502 (2014); 10.1063/1.4885047

Wave propagation in assemblies of cemented spheres

AIP Conf. Proc. 1542, 233 (2013); 10.1063/1.4811910

Numerical modeling of elastic waves in inhomogeneous anisotropic media using 3D-elastodynamic finite integration technique

AIP Conf. Proc. 1433, 487 (2012); 10.1063/1.3703233

Finite Element Modeling of Guided Wave Propagation in Plates

AIP Conf. Proc. 820, 118 (2006); 10.1063/1.2184519

\section{AIP $\mid$ APL Photonics}

APL Photonics is pleased to announce Benjamin Eggleton as its Editor-in-Chief

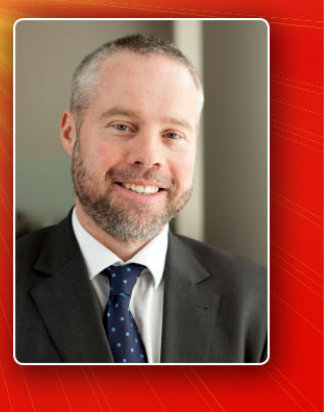




\title{
Wave propagation in cross-linked random fiber networks
}

\author{
Sahab Babaee, ${ }^{1}$ A. S. Shahsavari, ${ }^{2}$ Pai Wang, ${ }^{1}$ R. C. Picu, ${ }^{2}$ and Katia Bertoldi ${ }^{1,3}$ \\ ${ }^{1}$ School of Engineering and Applied Sciences, Harvard University, Cambridge, Massachusetts 02138, USA \\ ${ }^{2}$ Department of Mechanical, Aerospace and Nuclear Engineering, Rensselaer Polytechnic Institute, Troy, \\ New York 12180, USA \\ ${ }^{3}$ Kavli Institute, Harvard University, Cambridge, Massachusetts 02138, USA
}

(Received 23 July 2015; accepted 9 November 2015; published online 24 November 2015)

\begin{abstract}
We numerically investigate the propagation of small-amplitude elastic waves in random fiber networks. Our analysis reveals that the dynamic response of the system is not only controlled by its overall elasticity, but also by the local microstructure. In fact, we find that the longest fibersegment plays a key role in dynamics when the network is excited with waves of short wavelength. In this case, the Bloch modes are highly non-affine as the longest segments oscillate close to their resonances. Based on this observation, we predict the low frequency dispersion curves of random fiber networks. (O 2015 AIP Publishing LLC. [http://dx.doi.org/10.1063/1.4936327]
\end{abstract}

Disordered fibrous networks are ubiquitous and can be found in sound and thermal insulation systems, ${ }^{1}$ paper and paper products, ${ }^{2}$ filtration and liquid absorption systems, ${ }^{3}$ biological membranes and connective tissues, ${ }^{4-6}$ just to name a few. The structural behavior of these networks often plays a key role, motivating a deep interest in their mechanical response. $^{7}$ It is now well recognized that the mechanics of fibrous networks depends not only on the elastic properties of the constituent fibers but also on the network connectivity. ${ }^{1,2}$ Furthermore, it has been shown that the deformation transitions from affine to non-affine ${ }^{8-10}$ as the network density or fiber cross-sectional area decreases. ${ }^{11}$ However, despite the fact that fiber networks are often subjected to dynamic loadings, previous studies have primarily focused on the static behavior of random networks. ${ }^{12-16}$ Surprisingly, while the dynamic behavior of periodic beam networks has recently attracted considerable interest, ${ }^{17-20}$ the propagation of elastic waves in random fibrous networks has not yet been studied.

By contrast, the propagation of elastic waves in random spring networks ${ }^{23,24}$ and disordered packing of particles ${ }^{25-27}$ have been extensively studied. In particular, it has been shown that close to the jamming point (defined as the point where disordered matter transition from a flowing to a rigid state) such systems are anomalous in that they exhibit an excess of low frequency modes, and they do not appear to exhibit any ordinary continuum behavior. ${ }^{25-27}$ However, the response of random fiber networks, in which the bending deformation mode of fibers is taken into account (i.e., networks of beams), is significantly different, since such systems are rigid for average coordination numbers, $\bar{z}$, below the jamming point (i.e., for $\bar{z}<4$ ). ${ }^{26}$

In this letter, we investigate numerically the propagation of small-amplitude elastic waves in elastically stable random fibrous networks with $3<\bar{z}<4$, focusing on the first and second modes. We consider two-dimensional random networks composed of cylindrical fibers of length $L_{0}$, and radius $r$ made of an isotropic, linearly elastic material with Young's modulus $E_{f}$, shear modulus $G_{f}$, and density $\rho_{f}$. The networks are generated by depositing randomly $N$ fibers of equal length $L_{0}$ in a periodic unit cell of dimensions $a \times a$. Crosslinks (welded joints) are then introduced at all points where the fibers intersect, resulting in a Poisson distribution for the lengths of fiber-segments. ${ }^{28,29}$

It has been shown that the key parameters governing the static response of such fiber networks are the network density, $\rho=N L_{0} / a^{2}$, and the ratio between bending and axial stiffness of fibers, $l_{b}=\sqrt{\left(E_{f} I_{f}\right) /\left(E_{f} A_{f}\right)}=r / 2$ (where $I_{f}$ and $A_{f}$ denote the moment of inertia and the cross-sectional area of the fibers, respectively). ${ }^{11,28} \mathrm{We}$ choose the non-dimensional system parameters $\bar{\rho}=\rho L_{0}, \bar{r}=r / L_{0}=2 l_{b} / L_{0}, \bar{\lambda}=\lambda / L_{0}$, and $\bar{\omega}=\omega \bar{r} / \omega_{0}$ to present the results, where $\lambda$ and $\omega$ are the wavelength and frequency of the propagating elastic waves, respectively. Moreover, $\omega_{0}$ is the first resonance frequency of a fiber-segment of length $L_{0}$ and radius $r$, assuming clamped boundary conditions at both ends (i.e., $\omega_{0}=22.37$ $\sqrt{\left.\left(E_{f} r^{2}\right) /\left(4 \rho_{f} L_{0}^{4}\right)^{30}\right)}$. Finally, since we will find that the dynamic response of the networks is controlled also by the length of their longest fiber-segment, $L_{\max }$, we introduce the dimensionless parameter $\bar{L}_{\max }=L_{\max } / L_{0}$.

To study the propagation of small-amplitude elastic waves in such networks, we perform frequency-domain wave propagation analysis within the finite element (FE) framework using the commercial package Abaqus/Standard. Models of the periodic unit cell are built using EulerBernoulli beam elements (Abaqus element type B23), and Bloch-type boundary conditions are applied to the edges of the unit cell ${ }^{31,32}$ (see Ref. 33 for details on the wave propagation analysis). We then calculate the dispersion relation $\omega=\omega(\mathbf{k})$ using the frequency-domain perturbation method.

We start by computing the density of vibrational states for a network with $\bar{\rho}=25$ and $\bar{r}=0.004$ (see Fig. 1(c)). The results reported in Fig. 1(a) are based on a total of $2500 \mathrm{k}$-space points uniformly distributed across the entire first Brillouin zone and are normalized by the number of $\mathbf{k}$-space points. In stark contrast with the case of a network of springs, whose density of state plot is dominated by a peak at $\omega \sim 0$ (see Fig. S2 in Ref. 33), the response of the fiber network is characterized by several peaks at non-zero frequencies. The first peak occurs at $\bar{\omega}=0.011$, which corresponds to the resonance frequency of the longest fibersegment $\bar{L}_{\max }=0.59$. 
(a)

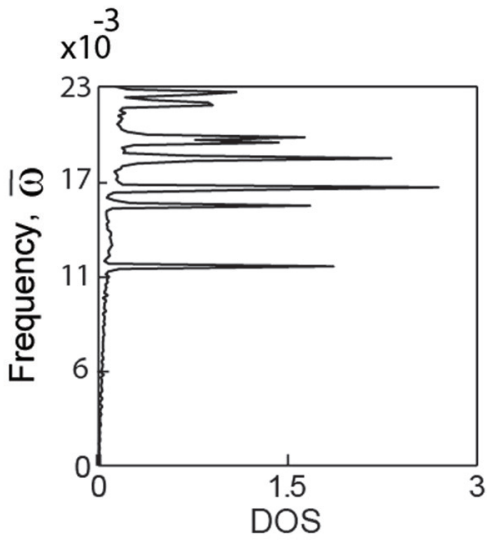

(b)

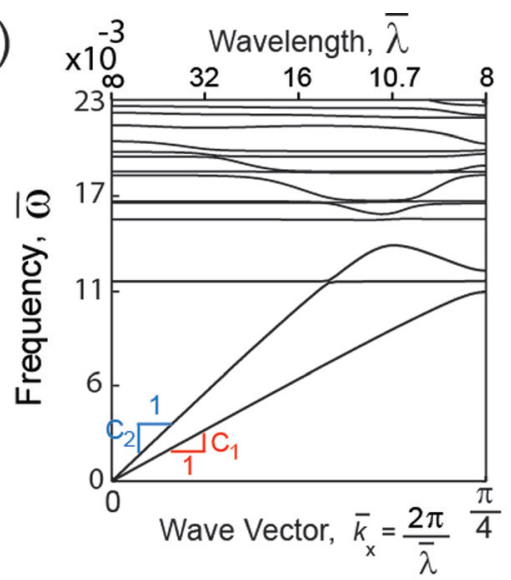

(c)

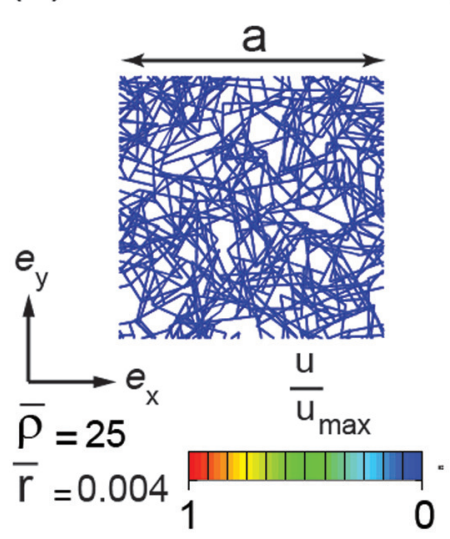

(d)

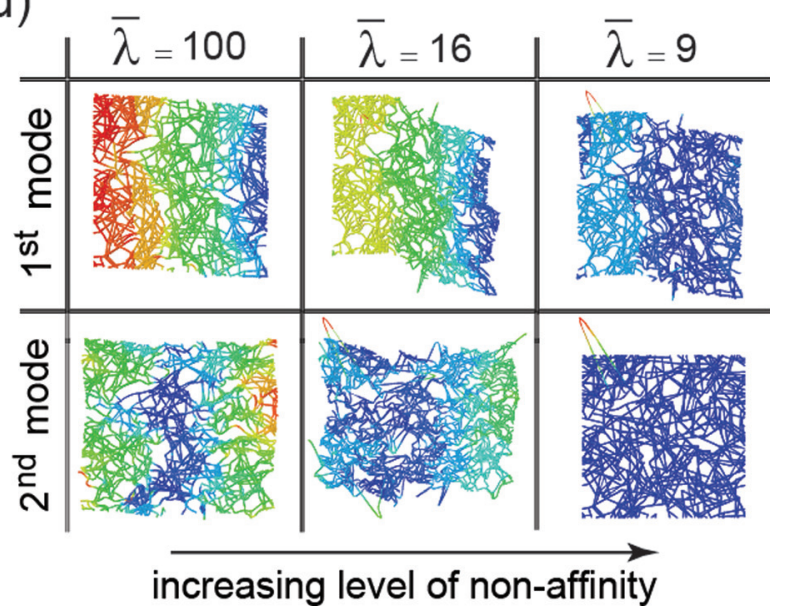

FIG. 1. (a) Density of vibrational states and (b) dispersion curves of a 2D crosslinked random network with $\bar{\rho}=25$ and $\bar{r}=0.004$ shown in (c). (d) The first (shear-like) and second (dilatationlike) Bloch modes for three different wavelengths of the propagating waves. Note that the hybridization of the folded shear-like mode with dilation-like mode observed at $\bar{\omega}=15$ in (b) (an artifact of Bloch-type boundary conditions) has already been observed for surface acoustic waves. ${ }^{21,22}$
To gain more insight into the dynamic behavior of the network, in Fig. 1(b), we plot its dispersion band diagram in an arbitrary direction (here chosen to be the horizontal direction $\mathbf{e}_{x}$ ). Similar to the case of an homogeneous linear elastic material, ${ }^{34}$ two bands emanate from the origin, corresponding to shear and dilatational waves propagating with speeds $c_{1}$ and $c_{2}$ in the long wavelength limit. However, as the wavelength decreases, the bands eventually reach the resonance frequency of the longest fiber-segment and flatten. ${ }^{35}$ It is important to note that this transition has a profound effect on the Bloch modes (see Fig. 1(d)). While in the long wavelength limit $(\bar{\lambda}=100$ in Fig. $1(\mathrm{~d}))$, both the first and second modes are characterized by low degree of non-affinity, for wavelength shorter than that of the transition $(\bar{\lambda}=9$ in Fig. $1(d)$ ), the vibrations are fully localized within the longest fiber-segment (see the red fiber with maximum displacement in the second mode), visually suggesting that that the level of non-affinity increases as the wavelength of the propagating waves decreases. ${ }^{36}$

To study the dynamic response of the fiber network in detail, first we investigate the response of the system in the long wavelength regime and determine the speeds $c_{1}$ and $c_{2}$ associated with the first (transverse) and second (longitudinal) modes as a function of the microstructural parameters of the network. Data for 115 random fiber networks with network densities $\bar{\rho}$ ranging from 12.5 to 25 , and $\bar{r}$ ranging from 0.00004 to 0.08 are presented in Fig. 2. To collapse the data onto a master curve, the same scaling parameter as in the static analysis of the network elasticity is used for the horizontal axis (i.e., $\left.w=\log _{10}\left[\bar{\rho}^{7}(\bar{r} / 2)^{2}\right)\right]^{37}$ ), and the wave speeds are normalized by the corresponding bulk properties of the fiber material (i.e., $\left(c_{f}\right)_{1}=\sqrt{G_{f} / \rho_{f}}$ and $\left.\left(c_{f}\right)_{2}=\sqrt{G_{f}\left(4 G_{f}-E_{f}\right) /\left[\rho_{f}\left(3 G_{f}-E_{f}\right)\right]^{34}}\right)$. As for the static case, the master plot has two well-defined regions. It has been shown that the transition between those two regions correlates with the transformation of the deformation field of the networks from non-affine to affine, as well as with the shift of energy storage from bending to axial. ${ }^{37}$ For small values of $w$, the slope of the master curve is $1 / 2$, indicating that the wave speeds of the networks are proportional to $\bar{\rho}^{3.5} \bar{r}$ in the long wavelength regime. Thus, the

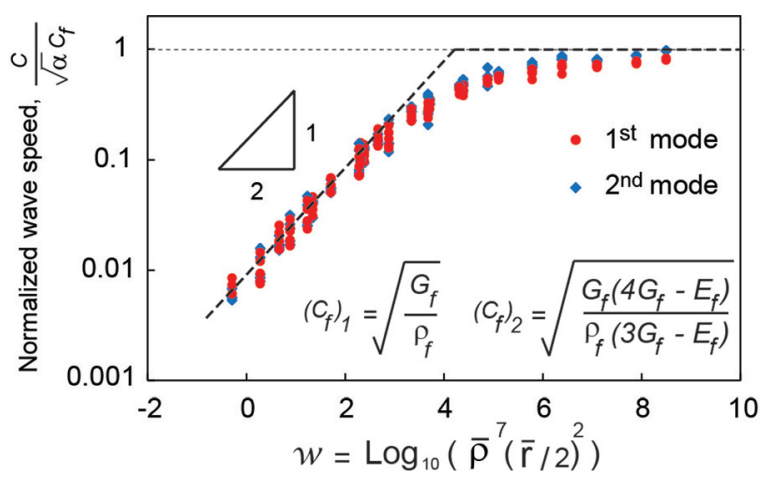

FIG. 2. Master plot providing the normalized wave speeds associated with the first and second modes as a function of system parameters. $\alpha=0.38$ is a non-dimensional constant that has the same value as in the analysis of network elasticity. ${ }^{37}\left(c_{f}\right)_{1}$ and $\left(c_{f}\right)_{2}$ are the transverse and longitudinal wave speeds for the fibers material, respectively. 
overall elastic modulus of networks, $E \sim c^{2} \bar{\rho} r^{2},{ }^{38}$ is proportional to $\bar{\rho}^{8} \bar{r}^{4}$, exactly as predicted by the static analysis. However, for $w>6$, all data converge to a horizontal asymptote, so that the long wavelength speeds of the networks become independent of $\bar{\rho}$ and $\bar{r}$, and $E \sim \bar{\rho} \bar{r}^{2}$, again in full agreement with the static analysis. ${ }^{37}$

Having determined the effect of the system parameters $\bar{\rho}$ and $\bar{r}$ on the long wavelength response, we proceed to investigate the effect of the dimensionless wavelength, $\bar{\lambda}$, on the propagation of the elastic waves. To this end, we calculate the dispersion bands for the networks and also investigate the effect of $\bar{\lambda}$ on their Bloch modes by directly measuring the evolution of their degree of non-affinity. Note that the deviation from affine deformation, denoted by $\triangle A_{j}$, for the $j$-th mode is defined as ${ }^{39}$

$$
\triangle A_{j}=\frac{1}{n U_{0}} \sum_{i=1}^{n}\left\|\mathbf{u}_{j}^{i}-\mathbf{u}_{a f f, j}^{i}\right\|,
$$

where $n$ is the number of the nodes in a full sinusoidal period, $U_{0}$ is the amplitude of the propagating wave, $\mathbf{u}_{j}^{i}$ is the displacement of the $i$-th node associated with the $j$-th mode, and $\mathbf{u}_{a f f, j}^{i}$ the corresponding values when the deformation is fully affine. For a wave propagating along the $\mathbf{e}_{x}$ direction, $\mathbf{u}_{a f f, 1}^{i}=U_{0} \sin \left[2 \pi\left(x_{i}-x_{0}\right) / \lambda\right] \mathbf{e}_{y}$, where $x_{i}$ identifies the coordinate in $\mathbf{e}_{x}$ direction of $i$-th node, $\mathbf{e}_{y}$ is a unit vector perpendicular to $\mathbf{e}_{x}$, and $x_{0}$ represents the phase of the mode.

In Figs. 3(a) and 3(b), we report the first dispersion band and the evolution of $\triangle A$ as a function of the normalized wavelength $\bar{\lambda}$ for two random networks characterized by the same values of $\bar{\rho}$ and $\bar{r}(\bar{\rho}=25$ and $\bar{r}=0.0004)$, but different lengths of the longest fiber-segment $\left(\bar{L}_{\text {max }}=0.55\right.$ and 0.9). ${ }^{40}$ Focusing on the dispersion bands (Fig. 3(a)), we see that the two networks are characterized by identical response in the long wavelength limit. We also find that in this region $\triangle A$ is not affected by $\bar{\lambda}$ (Fig. 3(b)). In fact, $\triangle A$ is the same as that calculated in the static case and is dictated by the overall elasticity of the system. ${ }^{13,28,41,42}$ By contrast, the wavelength $\bar{\lambda}^{*}$ and frequency $\omega^{*}$, at which the band flattens is found to be significantly affected by $\bar{L}_{\text {max }}$. For the network with $\bar{L}_{\max }=0.55$ (red line), we find that $\bar{\lambda}^{*}=22$ and $\bar{\omega}^{*}=0.0012$, while if $\bar{L}_{\max }=0.9$ (black line), $\bar{\lambda}^{*}=63$ and $\bar{\omega}^{*}=0.0005$. We also note that for $\bar{\lambda}<\bar{\lambda}^{*}$, $\triangle A$ suddenly rises (see Fig. 3(b)), reflecting the emergence of fully localized modes (i.e., see the second mode for $\bar{\lambda}=9$ in Fig. 1(d)).

Finally, the evolution of $\Delta A$ also reveals that the sharp transition at $\bar{\lambda}^{*}$ is preceded by another transition at $\bar{\lambda}^{\prime}$, at which $\triangle A$ gradually increases (note that $\bar{\lambda}^{\prime}$ is defined as the wavelength for which $\partial(\triangle A) / \partial \bar{\lambda}=0.2 \%)$. Inspection of the modes reveals that this increase of $\triangle A$ is induced by the (a)

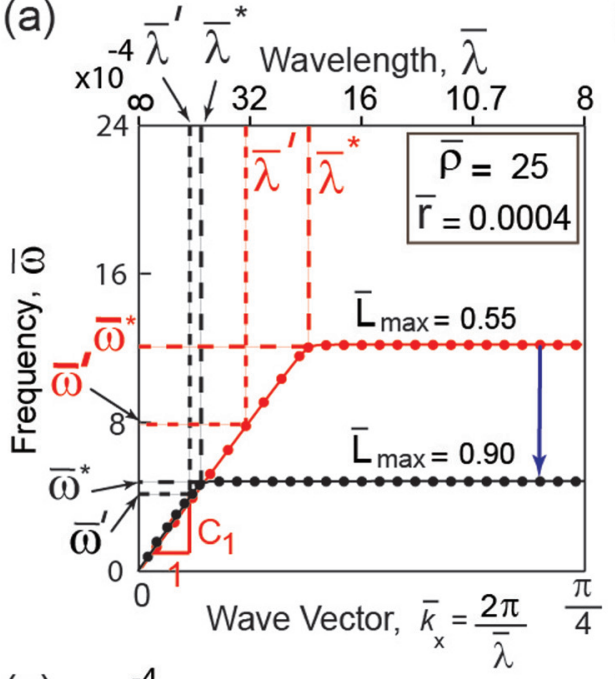

(c) $\times 10^{-4}$

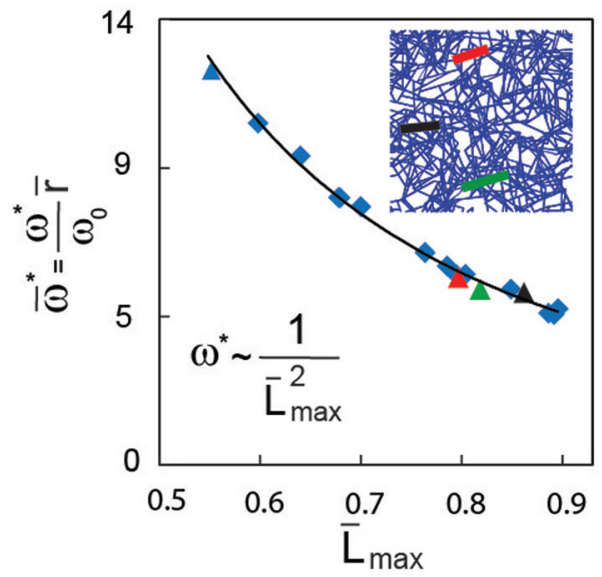

(b)

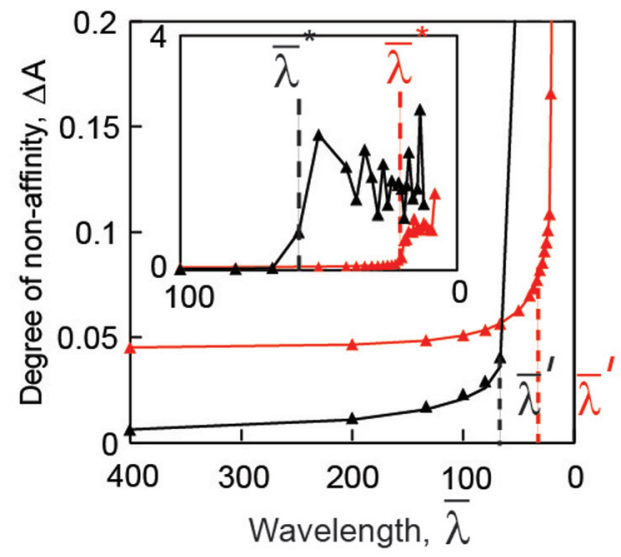

(d) $\times 10^{-4}$

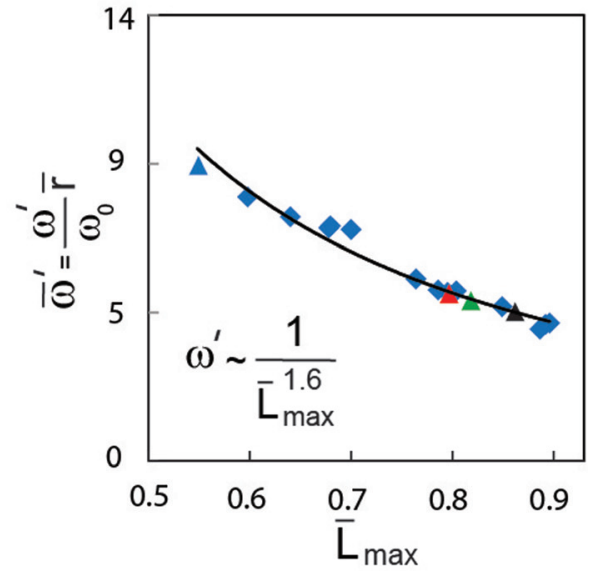

FIG. 3. (a) and (b) Effect of the longest fiber-segment, $\bar{L}_{\max }$, on (a) dispersion curves, and (b) degree of non-affinity as a function of $\bar{\lambda}$ for networks with $\bar{\rho}=25$ and $\bar{r}=0.0004$. (c) and (d) Effect of $\bar{L}_{\max }$ on transition frequencies (c) $\omega^{\prime}$, and (d) $\omega^{*}$, for different random networks (blue markers) with $\bar{\rho}=25$, $\bar{r}=0.0004$. The blue, red, green, and black triangular markers correspond to the exactly same network (shown as an inset in (c)), but with different $\bar{L}_{\max }$ attached to it (shown in the inset). 
gradual localization of the deformation within the longest fiber-segment of the network (i.e., see the first and second modes for $\bar{\lambda}=16$, and the first mode for $\bar{\lambda}=9$ in Fig. 1(d)). Importantly, we find that in the vicinity of this transition, the dispersion curve starts deviating from linearity (see Fig. S3 in Ref. 33), so that $\bar{\lambda}^{\prime}$ also identifies the threshold below which the fibrous network behaves as a dispersive wave medium. Interestingly, the results reported in Figs. 3(a) and 3(b) indicate that $\bar{\lambda}^{\prime}$ also is significantly affected by $L_{\max }$.

To fully understand the key role played by the longest fiber-segment in the dynamic response of random fiber networks, we calculate the characteristic frequencies, $\bar{\omega}^{\prime}=\omega^{\prime} \bar{r}$ / $\omega_{0}$ and $\bar{\omega}^{*}=\omega^{*} \bar{r} / \omega_{0}$, for 16 different networks characterized by the same system parameters $\bar{\rho}=25$ and $\bar{r}=0.0004$, but different values of $\bar{L}_{\max }$. The results reported in Fig. 3(c) show that $\bar{\omega}^{*}$ scales with $1 / \bar{L}_{\max }{ }^{2}$. This scaling law derives directly from the expression for the resonance frequency of a fibersegment of length $L_{\max }$ (i.e., $\omega^{*}=22.37 \sqrt{\left(E_{f} I_{f}\right) /\left(\rho_{f} A_{f} L_{\max }^{4}\right)}$, since this frequency corresponds to fully localization of vibrations within the longest fiber-segment. Moreover, we interpret the transition frequency $\bar{\omega}^{\prime}$ as the onset of resonance of the longest fiber-segment, and expect that $\bar{\omega}^{\prime} / \bar{\omega}^{*}$ is affected by the network environment surrounding the fiber of length $L_{\max }$. In particular, we expect that $\bar{\omega}^{\prime}$ is always smaller than $\bar{\omega}^{*}$, and $\bar{\omega}^{\prime} / \bar{\omega}^{*} \rightarrow 1$ as $\bar{L}_{\max }$ increases (i.e., $L_{\max }$ becomes very large compared with the other segments of the network). Therefore, since $\bar{\omega}^{*}$ scales with $1 / \bar{L}_{\max }{ }^{2}$, it is expected that $\bar{\omega}^{\prime}$ scales with $1 / \bar{L}_{\max }{ }^{m}$ in which $m<2$. Fig. 3(d) shows $\bar{\omega}^{\prime}$ is proportional to $1 / \bar{L}_{\max }{ }^{1.6}$ for networks with $\bar{\rho}=25$ and $\bar{r}=0.0004$. The robustness of these dependencies is confirmed by the fact that when we attach a longer fiber of lengths $\bar{L}_{\max }=0.796$ (red triangular marker), $\bar{L}_{\max }=0.821$ (green triangular marker), or $\bar{L}_{\max }=0.861$ (black triangular marker) to the network with $\bar{L}_{\max }=0.55$ (blue triangular marker), $\bar{\omega}^{\prime}$ and $\bar{\omega}^{*}$ are considerably altered, but are still proportional to $1 / \bar{L}_{\max } 1.6$ and $1 / \bar{L}_{\max }{ }^{2}$, respectively.

So far, we focused on fiber networks with $\bar{\rho}=25$ and $\bar{r}=0.0004$, and showed that their long wavelength response is purely governed by these two parameters, while $\bar{L}_{\max }$ plays an important role as $\bar{\lambda}$ is reduced, and strongly affects the wavelength $\bar{\lambda}^{*}$ at which the vibrations localize within a single fiber. However, it is important to recognize that $\bar{\lambda}^{*}$ is not only controlled by $\bar{L}_{\text {max }}$, but also by $\bar{\rho}$ and $\bar{r}$. In fact, $\bar{\lambda}^{*}$ is determined by the intersection between the flat band at $\bar{\omega}^{*}$ (which is determined by $\bar{r}$ and $\bar{L}_{\text {max }}$ ), and the band with wave speed $c_{1}$ in the long wavelength regime (which is controlled by $\bar{\rho}$ and $\bar{r}$, as predicted in Fig. 2).

To better understand these dependencies, in Fig. 4(a), we focus on networks with $\bar{r}=0.0004$ and $0.55<\bar{L}_{\max }$ $<0.9$, and determine $\bar{\lambda}^{*}$ for $\bar{\rho}=25,20$, and 12.5. Note that, for the sake of simplicity, $\bar{\lambda}^{*}$ is predicted without accounting for the deviation of the shear-dominated band from linearity occurring for $\bar{\lambda}<\bar{\lambda}^{\prime}$, since this deviation does not alter the trends discussed here. All networks considered in Fig. 4(a) are characterized by the same range of possible values for $\bar{\omega}^{*}$ (grey shaded region), while $c_{1}$ increases as $\bar{\rho}$ increases (according to the master plot in Fig. 2). Consequently, the range of possible values for $\bar{\lambda}^{*}$ moves to longer wavelengths
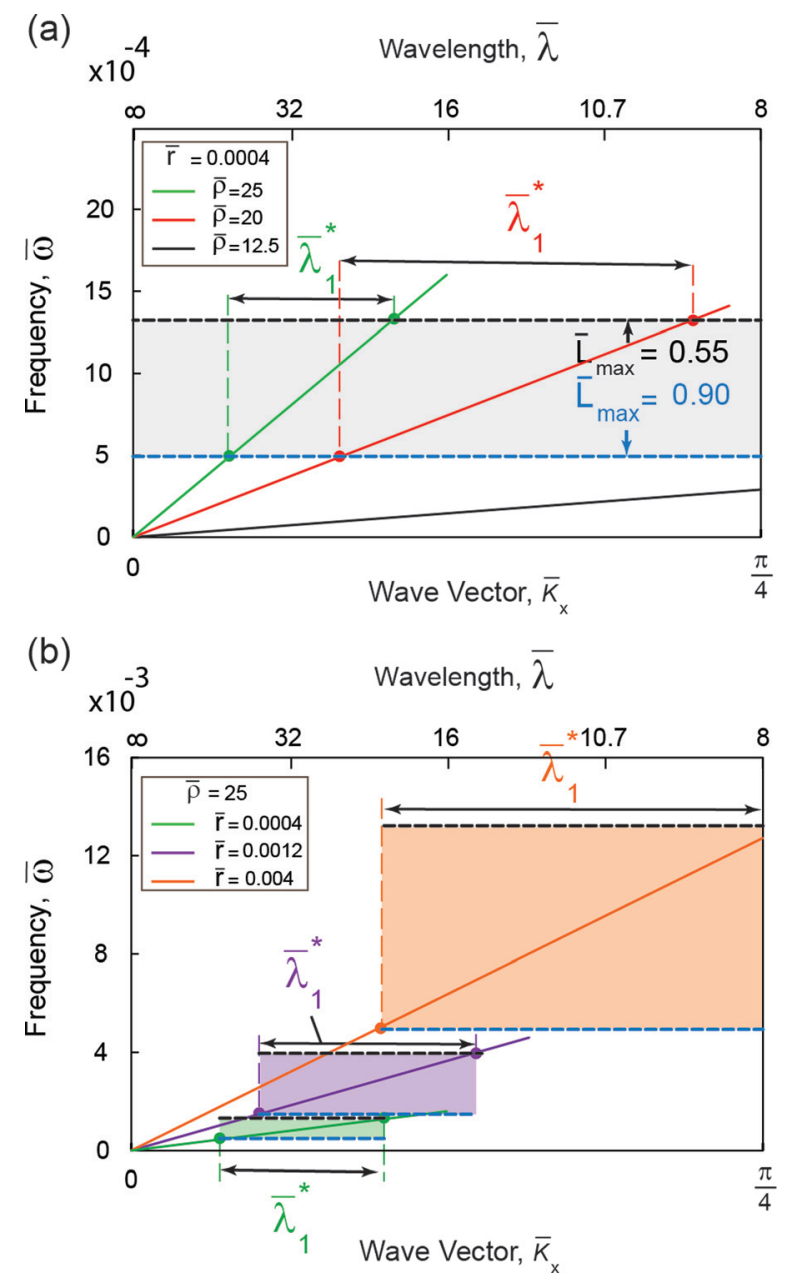

FIG. 4. Effects of (a) $\bar{\rho}$, and (b) $\bar{r}$ on the wavelength $\bar{\lambda}^{*}$, at which the vibrations localize within a single fiber for the first mode. The shaded areas indicate the range of resonance frequencies for networks with $0.55<\bar{L}_{\max }<0.9$.

as $\bar{\rho}$ increases. Note that such effect of $\bar{\rho}$ on $\bar{\lambda}^{*}$ is counterintuitive. In fact, we would expect that for larger values of $\bar{\rho}$, waves of shorter wavelength are needed to trigger localized modes, since according to static behavior of random networks,${ }^{11}$ both overall stiffness and level of affinity of the system increase monotonically with $\bar{\rho}$.

On the other hand, if $\bar{\rho}$ remains constant and $\bar{r}$ increases, the range of possible values for $\bar{\omega}^{*}$ increases quadratically, as illustrated in Fig. 4(b). Since $c_{1}$ increases at most linearly as $\bar{r}$ increases (see master plot in Fig. 2), the slope of the shear band in the long wavelength regime increases slower than $\bar{\omega}^{*}$ limits. Hence, in networks with larger $\bar{r}$, highly localized modes are more likely triggered at shorter wavelengths, as illustrated in Fig. 4(b).

In summary, our analyses reveal that the dynamic response of the networks depends not only on their microstructural parameters, but also on the length of the longest fiber-segment and the wavelength of the propagating waves. Furthermore, we found that that the propagation of the elastic waves through the system is characterized by three regimes: (i) for long wavelengths, the dynamic response of the system is fully controlled by the overall elasticity of the networks and is non-dispersive; (ii) for intermediate wavelengths, the networks behave as dispersive wave media and the non-affinity level of the Bloch modes increases 
gradually; (iii) in the short wavelengths regime, the Bloch modes are highly non-affine with all deformation localized within the longest fiber-segment of the network. We also note that in this study, we focused on the dynamic response of $2 \mathrm{D}$ networks, while the study of more realistic $3 \mathrm{D}$ systems (whose behavior has been found to be qualitatively similar to that of $2 \mathrm{D}$ networks for the static case $\mathrm{c}^{7,9}$ ) will be the subject of future investigations. Our results not only shed light on the propagation of elastic waves in random fibrous networks, but also open avenues for the design of fiber networks for applications in which the structure is exposed to mechanical vibrations.

This work has been supported by Harvard MRSEC through Grant No. DMR-1420570 and by NSF through Grant Nos. CMMI-1120724 and CMMI-1149456 (CAREER). K.B. acknowledges start-up funds from the Harvard School of Engineering and Applied Sciences and the support from the Kavli Institute and Wyss Institute at Harvard University.

${ }^{1}$ A. Kabla and L. Mahadevan, J. R. Soc., Interface/R. Soc. 4, 99 (2007).

${ }^{2}$ R. C. Picu, Soft Matter 7, 6768 (2011).

${ }^{3}$ K. K. Chawla, Fibrous Materials (Cambridge University Press, Cambridge, New York, 1998).

${ }^{4}$ K. E. Kasza, A. C. Rowat, J. Liu, T. E. Angelini, C. P. Brangwynne, G. H. Koenderink, and D. A. Weitz, Curr. Opin. Cell Biol. 19, 101 (2007).

${ }^{5}$ D. A. Fletcher and R. D. Mullins, Nature 463, 485 (2010).

${ }^{6}$ C. Storm, J. J. Pastore, F. C. MacKintosh, T. C. Lubensky, and P. A. Janmey, Nature 435, 191-194 (2005).

${ }^{7}$ C. P. Broedersz and F. C. MacKintosh, Rev. Mod. Phys. 86, 995 (2014).

${ }^{8} \mathrm{An}$ affine deformation is one where a macroscopic deformation applied on a network is translated uniformly to the microscopic level. On the contrary, for a non-affine deformation, the microscopic deformation deviates from the far field one.

${ }^{9}$ D. Head, A. Levine, and F. MacKintosh, Phys. Rev. E 68, 061907 (2003).

${ }^{10}$ B. DiDonna and T. Lubensky, Phys. Rev. E 72, 066619 (2005).

${ }^{11}$ A. Shahsavari and R. C. Picu, Phys. Rev. E 86, 011923 (2012).

${ }^{12}$ C. P. Broedersz, X. Mao, T. C. Lubensky, and F. C. MacKintosh, Nat. Phys. 7, 983 (2011).

${ }^{13}$ C. Heussinger and E. Frey, Phys. Rev. Lett. 97, 105501 (2006).

${ }^{14}$ M. Wyart, H. Liang, A. Kabla, and L. Mahadevan, Phys. Rev. Lett. 101, 215501 (2008).

${ }^{15}$ D. Head, A. Levine, and F. MacKintosh, Phys. Rev. Lett. 91, 108102 (2003).

${ }^{16}$ E. Conti and F. MacKintosh, Phys. Rev. Lett. 102, 088102 (2009).

${ }^{17}$ M. Ruzzene, F. Scarpa, and F. Soranna, Smart Mater. Struct. 12, 363 (2003).

${ }^{18}$ A. S. Phani, J. Woodhouse, and N. A. Fleck, J. Acoust. Soc. Am. 119, 1995 (2006).

${ }^{19}$ P. Wang, F. Casadei, S. H. Kang, and K. Bertoldi, Phys. Rev. B 91, 020103 (2015).

${ }^{20}$ D. Mousanezhad, S. Babaee, R. Ghosh, E. Mahdi, K. Bertoldi, and A. Vaziri, Phys. Rev. B 92, 104304 (2015).
${ }^{21}$ A. A. Maznev, Phys. Rev. B 78, 155323 (2008).

${ }^{22}$ I. A. Veres, D. M. Profunser, A. A. Maznev, A. G. Every, O. Matsuda, and O. B. Wright, New J. Phys. 14, 123015 (2012).

${ }^{23}$ M. Kellomäki, J. A. ström, and J. Timonen, Phys. Rev. Lett. 77, 2730 (1996).

${ }^{24}$ Y. Cai and M. F. Thorpe, Phys. Rev. B 40, 10535 (1989).

${ }^{25}$ A. J. Liu and S. R. Nagel, Annu. Rev. Condens. Matter Phys. 1, 347 (2010).

${ }^{26}$ M. van Hecke, J. Phys.: Condens. Matter 22, 033101 (2010).

${ }^{27}$ M. Wyart, S. R. Nagel, and T. A. Witten, Europhys. Lett. 72, 486 (2005).

${ }^{28}$ J. Wilhelm and E. Frey, Phys. Rev. Lett. 91, 108103 (2003).

${ }^{29}$ Note that to ensure that the response of the networks in the long wavelength regime does not vary significantly with the size of the unit cell $a$, the fiber length is chosen such that $L_{0} / a=0.25 .^{37}$ Importantly, we will see that this choice guarantees that the response in the short wavelength regime is also not affected by the size of the unit cell. In fact, our analysis indicates that in the short wavelength regime, the deformation is localized in the vicinity of the longest fiber-segment, which is shorter than $L_{0}$. Therefore, by choosing $a=4 L_{0}$, we ensure that the size of the system is large enough to contain the subdomain in which the deformation is localized.

${ }^{30} \mathrm{~W}$. Thomson and M. Dahleh, Theory of Vibration with Applications, 5th ed. (Prentice Hall, 1998).

${ }^{31}$ P. Wang, J. Shim, and K. Bertoldi, Phys. Rev. B 88, 014304 (2013).

${ }^{32}$ S. Babaee, P. Wang, and K. Bertoldi, J. Appl. Phys. 117, 244903 (2015).

${ }^{33}$ See supplementary material at http://dx.doi.org/10.1063/1.4936327 for details on the wave propagation analysis, density of vibrational states, and dynamic response of finite-size domains.

${ }^{34}$ M. Maldovan and E. L. Thomas, Periodic Materials and Interference Lithography: For Photonics, Phononics and Mechanics (Wiley-VCH Verlag GmbH and Co. KGaA, 2009).

${ }^{35}$ Note that although flattening of the dispersion bands induced by local resonance has been already reported in many studies, here it is achieved without embedding additional resonating components, since the fibers themselves act as resonators.

${ }^{36}$ Note that although in this study we investigate the dynamic response of 2D random fiber networks by focusing on a sufficiently large unit cell and applying Bloch-type boundary conditions, qualitatively the same behavior is found when we consider finite-size domains and calculate their steadystate dynamic linearized response to harmonic excitation (see Ref. 33 for details). In particular, also for finite-size domains, we find that localization happens when the frequency of the propagating waves is in the vicinity of the natural frequency of the longest fiber-segment, indicating that this is not an artifact introduced by Bloch-type boundary conditions (i.e., it is not caused by constructive scattering from neighboring unit cells).

${ }^{37}$ A. S. Shahsavari and R. C. Picu, Int. J. Solids Struct. 50, 3332 (2013).

${ }^{38}$ The density of matter in $2 \mathrm{D}$ fibrous networks is given by $\rho_{\text {mat }}=\rho_{f}$ $\left(N L_{0} A_{f}\right) / a^{2}=\rho_{f} \rho A_{f}=\rho_{f} \rho\left(\pi r^{2}\right)$, so that $c \sim \sqrt{E / \rho_{\text {mat }}} \sim \sqrt{E /\left(\rho r^{2}\right)}$.

${ }^{39}$ P. Onck, T. Koeman, T. van Dillen, and E. van der Giessen, Phys. Rev. Lett. 95, 178102 (2005).

${ }^{40}$ Note that $\bar{L}_{\max }=0.55$ and $\bar{L}_{\max }=0.9$ are the lower and upper bounds for $\bar{L}_{\max }$ based on 25000 random networks we generated with $\bar{\rho}=25$ and $L_{0} / a=0.25$.

${ }^{41}$ M. L. Gardel, J. H. Shin, F. C. MacKintosh, L. Mahadevan, P. Matsudaira, and D. A. Weitz, Science 304, 1301 (2004).

${ }^{42}$ E. Huisman, T. van Dillen, P. Onck, and E. Van der Giessen, Phys. Rev. Lett. 99, 208103 (2007). 


\title{
Supplementary Materials for Wave Propagation in Cross-Linked Random Fiber Networks
}

\author{
Sahab Babaee, ${ }^{1}$ A. S. Shahsavari, ${ }^{2}$ Pai Wang, ${ }^{1}$ R. C. Picu, ${ }^{2}$ and Katia Bertoldi ${ }^{1,3}$ \\ ${ }^{1}$ School of Engineering and Applied Sciences, Harvard University, Cambridge, Massachusetts 02138, USA \\ ${ }^{2}$ Department of Mechanical, Aerospace and Nuclear Engineering, \\ Rensselaer Polytechnic Institute, Troy, NY 12180, USA \\ ${ }^{3}$ Kavli Institute, Harvard University, Cambridge, Massachusetts 02138, USA
}

(Dated: November 9, 2015)

\section{WAVE PROPAGATION ANALYSIS}

The propagation of elastic waves within cross-linked random fiber networks is investigated numerically by considering $2 \mathrm{D}$ periodic unit cells spanned by the lattice vectors $\mathbf{a}_{1}=[a, 0]$ and $\mathbf{a}_{2}=[0, a]$, as shown in Fig. S1(a).

(a)

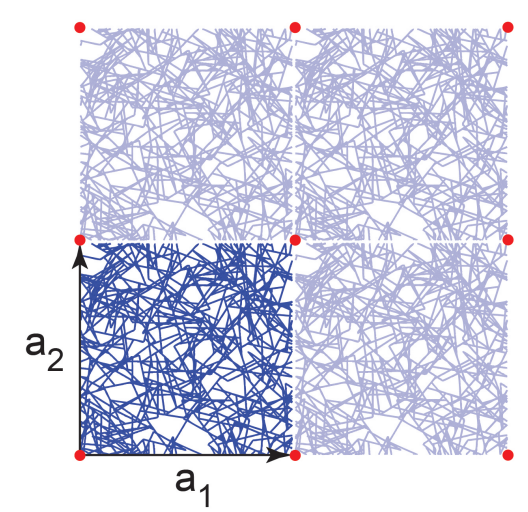

(b)

\section{Reciprocal space}

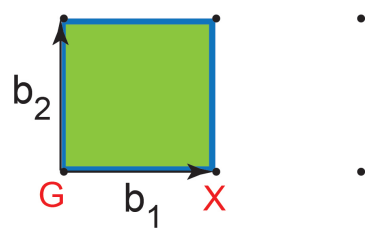

FIG. S1: (a) 2D periodic unit cell spanned by the lattice vectors $\mathbf{a}_{1}=[a, 0]$ and $\mathbf{a}_{2}=[0, a]$. (b) Corresponding point lattice and first Brillioun zone (green area) in the reciprocal space.

In order to obtain the dispersion relation of the propagating waves in the random fiber networks, frequency domain wave propagation analysis are performed on the unit cells. Bloch-type boundary conditions of the form

$$
\mathbf{u}(\mathbf{x}+\mathbf{r})=\mathbf{u}(\mathbf{x}) \exp (i \mathbf{k} \cdot \mathbf{r}),
$$

are applied to the edges of the unit cell, where $\mathbf{u}$ and $\mathbf{x}$ denote the displacement and position of a point. Moreover, $\mathbf{r}$ is the distance between each pair of nodes periodically located on the boundaries and $\mathbf{k}$ is Bloch-wave vector. Since most commercial finite element packages do not support the complex-valued displacements introduced by (S1), following Aberg and Gudmundson [1], we split any complex-valued spatial function $\phi(\mathbf{x})$ into a real and an imaginary part

$$
\phi(\mathbf{x})=\phi(\mathbf{x})^{r e}+i \phi(\mathbf{x})^{i m} .
$$

The problem is then solved using two identical finite element meshes for the unit cell, one for the real part and the other for the imaginary part, coupled by

$$
\mathbf{u}^{r e}(\mathbf{x}+\mathbf{r})=\mathbf{u}^{r e}(\mathbf{x}) \cos (\mathbf{k} \cdot \mathbf{r})-\mathbf{u}^{i m}(\mathbf{x}) \sin (\mathbf{k} \cdot \mathbf{r})
$$

and

$$
\mathbf{u}^{i m}(\mathbf{x}+\mathbf{r})=\mathbf{u}^{r e}(\mathbf{x}) \sin (\mathbf{k} \cdot \mathbf{r})+\mathbf{u}^{i m}(\mathbf{x}) \cos (\mathbf{k} \cdot \mathbf{r}) .
$$

Note that Eqs. (S3) and (S4) are implemented into Abaqus/Standard via multi-point constraints [2].

Focusing on the propagation of small amplitude waves, we then calculate the dispersion relation $\omega=\omega(\mathbf{k})$ using the frequency domain perturbation method. In particular, the dispersion band diagram reported in Fig. 3(a) is obtained by choosing $\mathbf{k}=\kappa_{x} \mathbf{e}_{x}$ and considering fifty uniformly-spaced points in the interval $\kappa_{x} \in[0, \pi / a]$. 


\section{DENSITY OF VIBRATIONAL STATES (DOS) FOR NETWORKS OF SPRINGS AND BEAMS}

In Fig. S2 we report the density of vibrational states (DOS) for a $2 \mathrm{D}$ cross-linked random fiber network (beam network - Fig. S2(a)), and the corresponding spring network (Fig. S2(b)) with $\bar{\rho}=25$ and $\bar{r}=0.004$. The results are calculated based on a total of $2500 \mathrm{k}$-space points uniformly-distributed across the entire first Brillouin zone and are normalized by the number of $\mathbf{k}$-space points. While the response of the spring network is dominated by a peak at $\omega \sim 0$, for the fiber beam network the response is characterized by several peaks at non-zero frequencies.

(a)

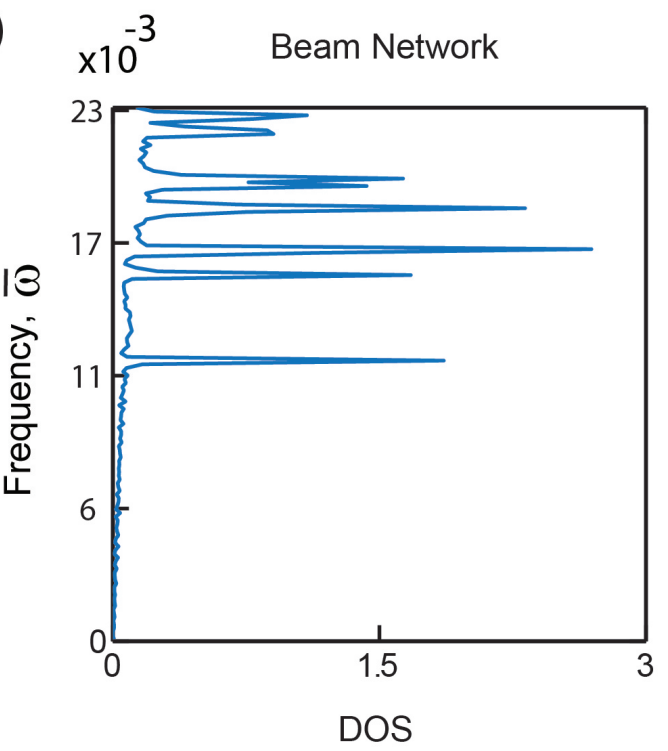

(b)

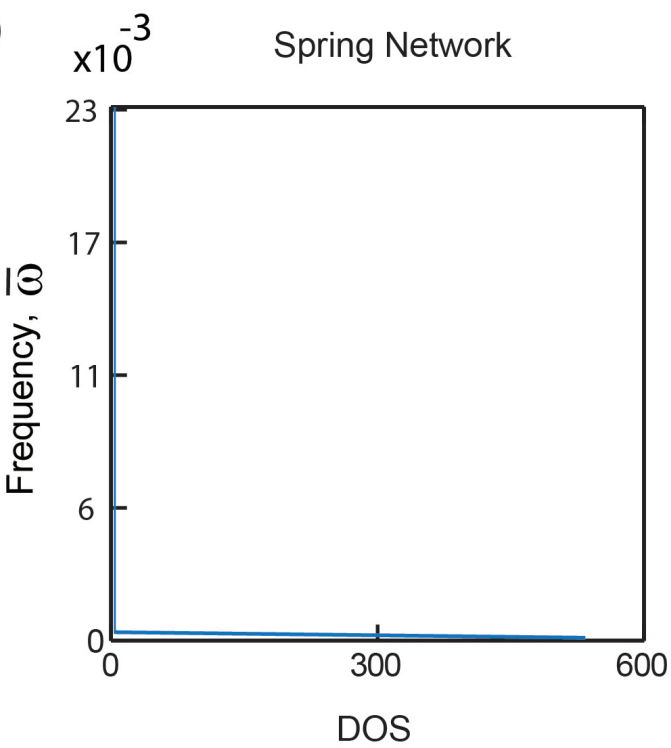

FIG. S2: Density of vibrational states for (a) a 2D cross-linked random fiber network (beam network), and (b) the corresponding spring network. Both networks are characterized by $\bar{\rho}=25$ and $\bar{r}=0.004$. 


\section{GROUP VELOCITIES}

In Fig. S3 we report the first dispersion band $(\bar{\omega})$ and the evolution of the group velocity $\left(d \bar{\omega} / d \bar{\kappa}_{x}\right)$ as a function of the wave vector for two random networks characterized by the same values of $\bar{\rho}$ and $\bar{r}(\bar{\rho}=25$ and $\bar{r}=0.0004)$, but different lengths of the longest fiber-segment $\left(\bar{L}_{\max }=0.55\right.$ and 0.9 in Figs. S3(a) and S3(b), respectively). In the plots we also show the transition wavelengths $\bar{\lambda}^{*}$ and $\bar{\lambda}^{\prime}$.

As described in the main text, $\bar{\lambda}^{*}$ is defined as the wavelength at which the band flattens. Therefore, we find that $d \bar{\omega} / d \bar{\kappa}_{x}$ sharply decreases to zero at $\bar{\lambda}^{*}$.

Moreover, our analysis indicates that the sharp transition at $\bar{\lambda}^{*}$ is preceded by another transition at $\bar{\lambda}^{\prime}$, at which the degree of non-affinity $\triangle A$ gradually increases (note that $\bar{\lambda}^{\prime}$ is defined as the wavelength for which $\partial(\triangle A) / \partial \bar{\lambda}=0.2 \%$ - see Fig. 3(b) in the main text). Importantly, the results reported in Fig. S3 reveals that at $\bar{\lambda}^{\prime}$ the dispersion curve starts deviating from linearity (as $d \bar{\omega} / d \bar{\kappa}_{x}$ starts deviating from a constant value - see insets in Fig. S3), so that this transition defines the threshold below which the fibrous network behaves as a dispersive wave medium.

(a) $\times 10^{-4}$

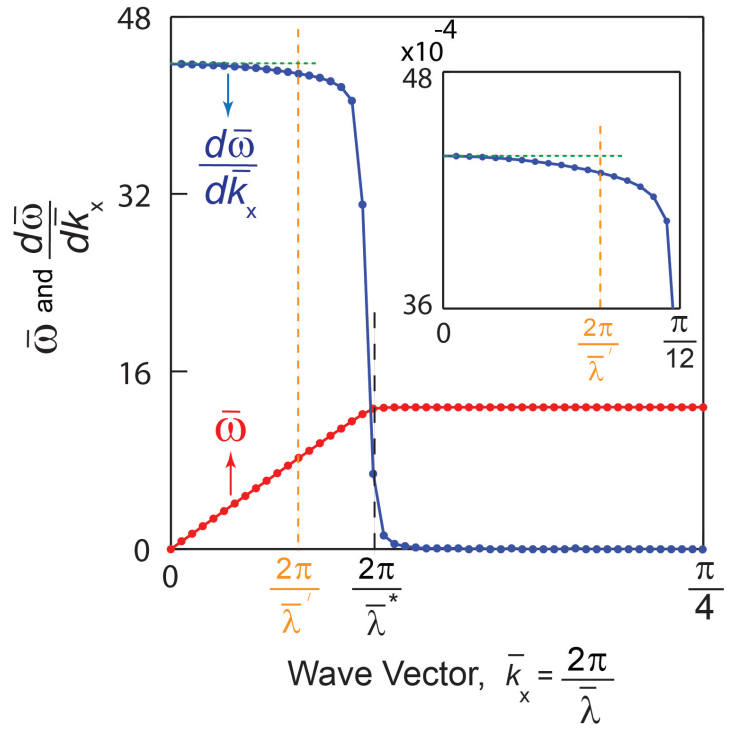

(b)

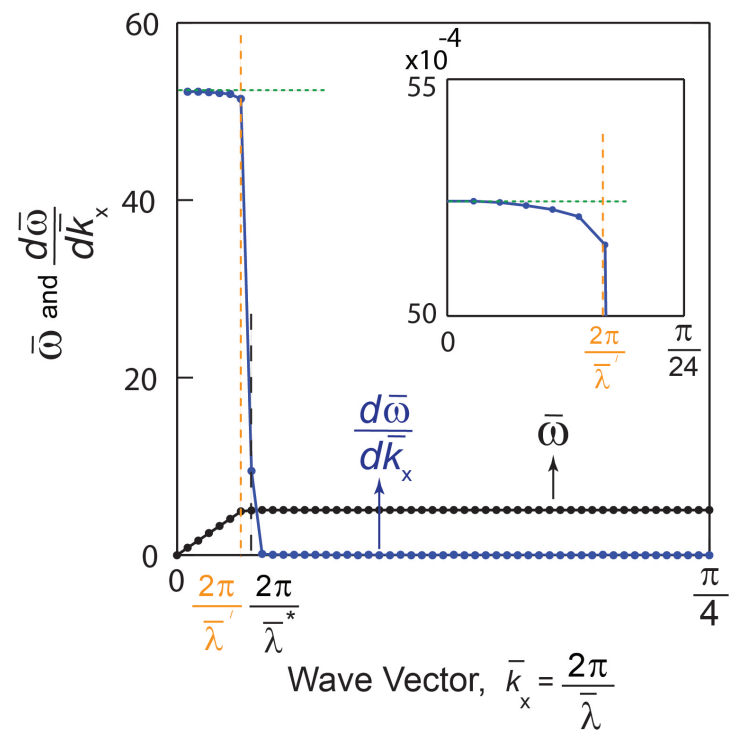

FIG. S3: First dispersion band $(\bar{\omega})$ and the evolution of the group velocity $\left(d \bar{\omega} / d \bar{\kappa}_{x}\right)$ as a function of wave vector for two random networks characterized by $\bar{\rho}=25$ and $\bar{r}=0.0004$, and different lengths of the longest fiber-segment $\left((\mathrm{a}) \bar{L}_{\text {max }}=\right.$ 0.55 and (b) $\left.\bar{L}_{\max }=0.9\right)$. The insets show the zoom-in views of $d \bar{\omega} / d \bar{\kappa}_{x}$ in the vicinity of $\bar{\lambda}^{\prime}$. 


\section{DYNAMIC RESPONSE FOR FINITE-SIZE RANDOM FIBER NETWORKS}

In this study we investigate the dynamic response of $2 \mathrm{D}$ random fiber networks by focusing on a sufficiently large unit cell and applying Bloch-type boundary conditions. To clarify the effect of such quasi-periodic boundary conditions, here we consider 2D random fiber networks of finite-size and calculate their steady-state dynamic linearized response to harmonic excitation. In this set of analyses, we excite the network by applying an harmonic displacement (i.e. $\left.A_{\text {in }} \cos (\omega t)\right)$ to all the nodes on the left edge of the model and calculate the displacement field distribution (i.e. deformation modes) over the frequency ranges of interest.

In Fig. S4 we report the deformation modes for different values of $\omega$ for the same network shown in Fig. 1 of the main text (characterized by $\bar{\rho}=25$ and $\bar{r}=0.004$ ) when considered as a finite-size domain. The results indicate that in the low-frequency (i.e. in the long wavelength limit) the vibrations are diffuse through the system (see snapshot in Fig. S4 for $\bar{\omega}=1.3 \times 10^{-3}$ ), as predicted by the Bloch modes of the same periodic network for $\bar{\lambda}=100$ reported in Fig. 1(d) of the main text. However, as the resonance frequency of the longest fiber $\left(\bar{\omega}_{\text {resonance }}=12.0 \times 10^{-3}\right)$ is approached, the deformation starts to be gradually localized within the longest fiber-segment, as highlighted by the red dashed circle in Fig. S4(b). For $\bar{\omega}=\bar{\omega}_{\text {resonance }}=12.0 \times 10^{-3}$ (i.e. at the resonance frequency of the longest fiber) the vibrations are fully localized within the longest fiber-segment (see fiber with maximum displacement highlighted by the red ellipse in Fig. S4(c)). Finally, the localization is weakened as the frequency gets further away from $\bar{\omega}_{\text {resonance}}$.

In summary, our finite-size results are in good agreement with those obtained by applying Bloch-type boundary conditions (see Bloch modes reported in Fig. 1(d) of the main text for the same network), validating the numerical approach used in this study.

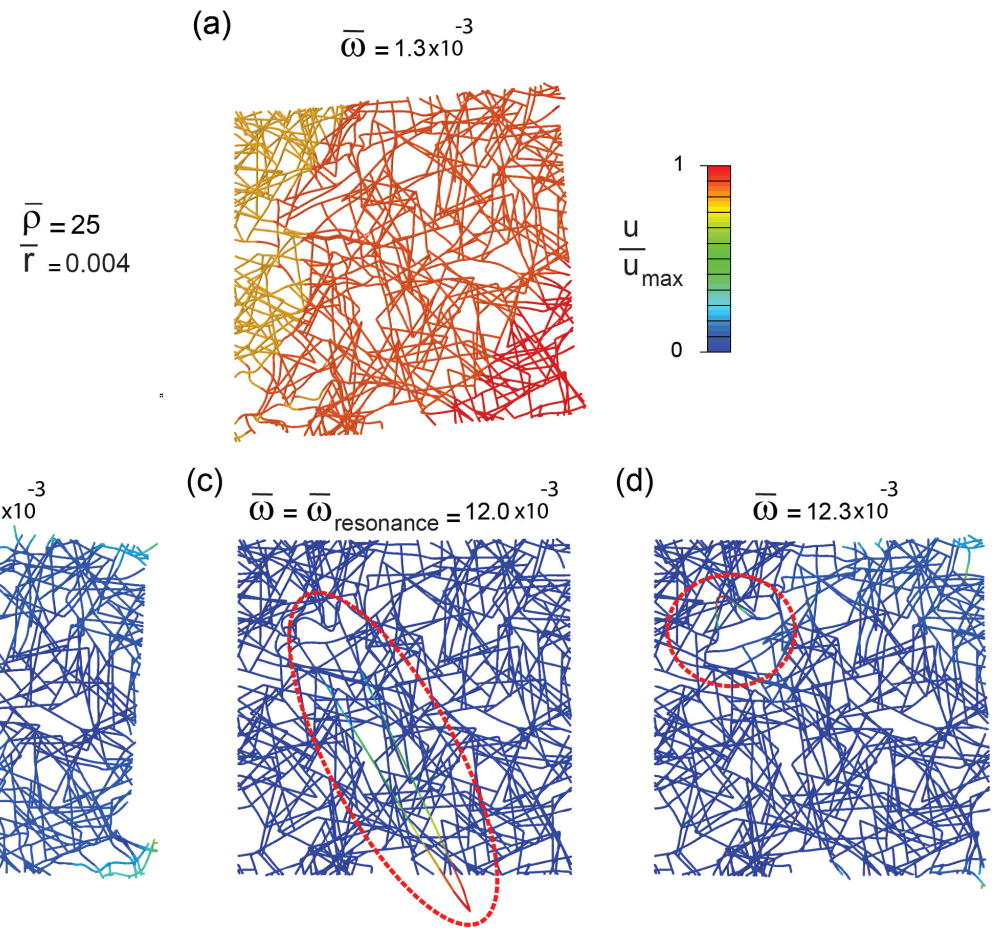

FIG. S4: Steady-state response of a finite size random network with $\bar{\rho}=25$ and $\bar{r}=0.004$. Deformation modes are shown at different excitation frequencies: (a) $\bar{\omega}=1.3 \times 10^{-3}$ (low frequency), (b) $\bar{\omega}=11.7 \times 10^{-3}$ (in the vicinity of the resonance frequency of the longest fiber), (c) $\bar{\omega}=\bar{\omega}_{\text {resonance }}=12.0 \times 10^{-3}$ (at the resonance frequency of the longest fiber) and (d) $\bar{\omega}=12.3 \times 10^{-3}$ (in the vicinity of the resonance frequency of the longest fiber). The red dashed ellipses highlight the deformation of the longest fiber. 
[1] M. Aberg and P. Gudmundson, The Journal of the Acoustical Society of America 102, 2007 (1997).

[2] P. Wang, J. Shim, and K. Bertoldi, Phys. Rev. B 88, 014304 (2013). 